\title{
On Translating UML Models into Graph Transformation Systems
}

\author{
Karsten Hölscher ${ }^{\mathrm{a}, 1}$ Paul Ziemann ${ }^{\mathrm{a}, 2}$ Martin Gogolla ${ }^{\mathrm{a}, 3}$ \\ ${ }^{a}$ Department of Computer Science \\ University of Bremen \\ Bremen, Germany
}

\begin{abstract}
In this paper we present a concept of a rigorous approach that provides a formal semantics for a fundamental subset of UML. This semantics is derived by translating a given UML model into a graph transformation system, allowing modelers to actually execute their UML model. The graph transformation system comprises graph transformation rules and a working graph which represents the current state of the modeled system. In order to support UML models which use OCL, we introduce a specific graph transformation approach that incorporates full OCL in the common UML fashion. The considered UML subset is defined by means of a metamodel similar to the UML 1.5 metamodel. The concept of a system state that represents the state of the system at a specific point in time during execution is likewise introduced by means of a metamodel. The simulated system run is performed by applying graph transformation rules on the working graph. The approach has been implemented in a research prototype which allows the modeler to execute the specified model and to validate the basic aspects of the model in an early software development phase.
\end{abstract}

Key words: Graph transformation, UML semantics, validation, CASE tool

\section{Introduction}

The Unifi ed Modeling Language (UML) has recently become a standard for the modeling of object-oriented software systems. It comprises a set of different diagram types, each of them describing particular aspects of software artifacts. The

\footnotetext{
ऋ Research partially supported by the EC Research Training Network SegraVis (Syntactic and Semantic Integration of Visual Modeling Techniques) and the project Abstract Implementation of and Documentation with UML (UML-AID) funded by the German Research Foundation (DFG).

1 Email: hoelschereinformatik.uni-bremen.de

2 Email: ziemanneinformatik.uni-bremen.de

3 Email: gogolla@informatik.uni-bremen.de

This is a preliminary version. The final version will be published in Electronic Notes in Theoretical Computer Science URL: www.elsevier.nl/locate/entcs
} 
syntax of these diagrams is described by means of a metamodel in [OMG03], denoted as class diagrams. Since the class diagram itself is defi ned in a cyclic way by the metamodel, the metamodel defi nition of UML diagrams can only be considered semi-formal. Furthermore the semantics of UML components is only expressed in natural language. In order to overcome the limitations of a purely graphical notation, the UML has been enhanced by the textual Object Constraint Language (OCL). The OCL is also semi-formally defi ned in [OMG03]. A formal syntax and semantics for UML class diagrams as well as OCL has been introduced in [Ric02], which is also included in the accepted OCL 2.0 submission to the OMG [BCI03]. This work presents a concept for obtaining a formal semantics not only for class diagrams but for further basic diagram types (use case, object, statechart and interaction diagrams) belonging to the UML standard 1.5. We stick to UML 1.5 but UML 2.0 likewise includes the UML concepts covered by us, albeit some details and the naming have been changed in some cases. In particular, UML 1.5 collaboration diagrams are called communication diagrams in UML 2.0. Graph transformation (cf. [Roz97], [EEKR99], [EKMR99]) is employed as the formal foundation of this new integrating semantics.

Our approach provides a framework for an automatic translation of a UML model into a graph transformation system. The UML model may comprise a subset of the diagram types mentioned above and may furthermore include OCL expressions. The graph transformation system consists of graph transformation rules and a socalled working graph, hence called system state graph. This graph represents the state of the modeled system at a given point of time. The changes of the system state during an execution of the model are simulated by the application of graph transformation rules on the system state graph. In this way a stepwise execution of the model can be simulated. As no formal semantics is given for the UML, the effects of the model execution rely on a number of assumptions, especially regarding the integration of the mentioned diagram types and their use in practice. We have enhanced the graph transformation foundation with OCL expressions. These expressions navigate the current system state and can be used as application conditions, which determine whether a certain rule may or may not be applied. OCL expressions can also be used to calculate new attribute values in the right-hand side of graph transformation rules. Additionally, OCL is used as query language for inspecting the current system state. The use of OCL as a textual notation also leads to the benefi $t$ of more compact graphs in most cases.

The representation of a UML model as a graph transformation system is used here to validate the system before actually implementing it. Employing graph transformation for the simulation of the modeled system has the benefi $t$ of a visualized system run. The simulation allows modelers to compare the behavior of the system with their expectations. Given a system state $\alpha$, they can easily gain an understanding of the actions that are possible in this state. Furthermore our concept also supports goal-oriented tests regarding the question whether a given state $\omega$ is reachable from state $\alpha$ (though this is generally undecidable as it is equivalent to the word problem for any kind of language). The approach also enables the modeler to check 
what states can be derived from the state $\alpha$. The integration of OCL allows for the checking of OCL invariants during system state evolutions. The modeler may test whether an invariant that holds in state $\alpha$ still holds in a derived system state $\omega$.

Currently, a prototypic validation system is implemented for our approach which generates the graph transformation rules for a given model and allows to interactively execute and visualize the modeled system. This prototype can be used by a modeler in an early stage of a software development process in order to acquire additional insight into the newly designed system.

A full formal and elaborated description of our concepts can be found in the PhD thesis [Zie05], where the integrated system specifi cation and the translation into a graph transformation system as well as the implemented prototype UGT are discussed: In the central part of that work, the translation of an integrated UML model into a graph transformation system is described in detail. The concept of system states, which are represented by graphs and transformed by graph transformation rules, is defi ned. The thesis explains how to derive the initial system state, which is the system state at the beginning of a system run, from the given object and statechart diagrams. The graph transformation rules are described subsequently. The work describes the rule for initiating use cases, which is independent of the UML model. Also independent of the model are the rules performing predefi ned operations like creating objects or setting attributes. The paper describes rules executing object operations, i.e., operations the user has declared in a class diagram and specifi ed by an interaction diagram. Use cases are treated as operations that do not belong to a class (use case operations), and therefore the rules realizing use cases are constructed in the same way. Nodes that are not needed anymore are deleted from the system state by the garbage collection rule. Finally, the work clarifi es the way class generalization and thus inheritance of attributes and operations are covered. For all details, we again refer to the original work [Zie05].

The structure of the rest of this paper is as follows. In the next section we present some related work, followed by a section in which the concepts of graph transformation we employ are introduced. The covered UML features of the model are presented and explained using a simple example in Section 4. Section 5 deals with the detailed description of the system state concept. An overview of the translation of the model into a graph transformation system is presented in Sect. 6 mostly by example, followed by a brief introduction to the fundamental architecture of the prototypic implementation. The paper closes with concluding remarks in Sect. 7.

\section{Related Work}

Since UML lacks a formal mathematical foundation, several works can be found that address this issue. Different formalisms are employed to provide a formal semantics for parts of UML. In [KC00] a translation of UML into a partial Object$\mathrm{Z}$ specifi cation is presented. The work in [CEK01,EK99,FELR98] discusses the specifi cation of UML semantics on the metamodel level. [Stö05] presents a formal semantics of UML Activities based on Petri-nets. Streams are employed as seman- 
tic domain of a UML model in $\left[\mathrm{BHH}^{+}\right.$97]. The works [EW01,Wie98] focus on high level semantics based on temporal and deontic logic, respectively. The xUML approach presented in [Sta01] defi nes a subset of UML for rigorous object-oriented modeling and provides an operational semantics for supported diagram types.

There are also several other works aiming at defi ning a semantics for parts of UML using graph transformation. In [KGKK02], an integrated semantics is given for a large part of UML. However, interaction diagrams and OCL are not considered. Their approach is extended with interaction diagrams on instance level in [GZK03]. Operations are still specifi ed by single rules, that is, all operations have to be atomic. More efforts exist considering isolated parts of UML. In [HS01], collaborations are translated into transformation rules, where collaborations are interpreted as visual queries using pattern matching. A formal semantics for UML statecharts is presented for example in [Kus01,Var02]. The Fujaba tool suite [FNTZ98] supports graphical object-oriented software design and automatic code generation from story diagrams. These diagrams combine behavioral UML diagrams and additional features. Additional approaches for consistency analysis of UML models can be found. In [EHKG02], given UML real time models are refi ned using graph transformation rules and their consistency is checked in the semantic domain of CSP. [And98] studies questions concerning the realization and the semantics of UML packages in connection with a graph-based tool. [TE00] addresses the consistency analysis between UML class and sequence diagrams based on graph transformation. Parts of the UML semantics have also been defi ned with Abstract State Machines (ASMs). Work includes the UML semantics defi nition for single diagram like statechart [BCR00b,CRS03] and activity diagrams [BCR00a] and the study of special aspects like constraints [FM04] or liveness [CF04]. Furthermore, ASMs have been used for the validation of UML models [SCH01] and the implementation of a UML virtual machine [SCH03]. However, a comprehensive integrating treatment of these different works seems to be missing.

All these works have in common, that they mostly address isolated parts of UML. We are not aware of an approach handling a collection of UML diagrams as presented in this work together with the integration of OCL. In particular the incorporation of use cases is new. In [SSO0] use cases are described precisely by so-called operation schemata including OCL pre- and postconditions but the connection to other UML diagrams is left open.

The main benefi $t$ of our approach is (A) the integrated coverage of a substantial part of the UML, (B) a minimal impedence mismatch between the original UML model and our semantical domain, in particular the system state and (C) the possibility to validate and test on the model level. As the system evolves, our system state graph changes accordingly, always in tight closeness to the actual visual UML model. The system state may be understood as a UML object diagram. Analogously to the system state, our rules, e.g., for statechart transitions, bear strong similarities to the original UML descriptions. The closeness between UML model and the semantical domain allows to give better feedback to the modeler because the modifi ed system state can again be understood as a UML description. Our approach also makes 
fewer assumptions regarding the semantics of the model as, for instance, other approaches and code generators have to make. In order to improve the quality of software, the application of code generators is an accepted means in the industrial software development. As direct tests of the model are not yet supported, the generated code is debugged. Should errors occur during tests, currently the generated code is changed. But in our approach, it is possible to directly perform tests on the level of the model without having to deal with generated code. Our approach also allows for an easier handling of future extensions and changes of the model, since only the model itself and not the generated code has to be changed.

\section{Graph Transformation}

Graphs are a frequently used means to visualize complex information, like the structure of computer networks or database designs. Graph transformation allows local changes on such a graph by applying graph transformation rules. A very obvious application domain for graph transformation is the fi eld of visual modeling, like UML. A model may be transformed into another model of the same visual language (e.g. refi nement or refactoring) or into a model of a different visual language (cf. MDA). A graph transformation system may also serve as a semantic domain, as is the case in our approach. Various well-studied graph transformation approaches can be found in the literature.

Basically a graph consists of a set of vertices, a set of edges, and mappings $s, t$ that assign a source and a target vertex to every edge. Furthermore vertices and edges are mapped into a given alphabet, providing edge and vertex labels. The label alphabet includes the invisible label, which is used for vertices or edges whenever they are supposed to carry no label.

Since we want to represent more sophisticated structures where objects may also contain attributes, we enhance this graph model with a concept for vertex attributation. Analogously to the presentation in [HKT02], data values are represented as special data value nodes, all of which are present in the attributed graph. The elements of the combined set of data value nodes and "usual" vertices will be called nodes. The source, target and label mappings then apply to the set of all nodes, where the data value nodes are labeled with their respective type. In our approach the data value nodes are the elements of the mathematical sets $\mathbb{Z}, \mathbb{R},\{$ true, false $\}$, $A^{*}$ (for a given alphabet $A$ ) representing the OCL types Integer, Real, Boolean, and String, respectively. An attribute of a vertex is then represented by an edge from that vertex to the corresponding data value node. This edge is labeled with the name of the attribute. Since the carrier sets are usually infi nite, data value nodes are omitted in pictures unless they have incoming edges. In this case we call the data value nodes visible.

In fi gures we employ a simplifi ed notation of vertex-attributed graphs by depicting them in a UML-like fashion. Vertices are depicted as rectangles with two compartments. In the upper compartment the vertex identifi er and its label can be found, separated by a colon. Here the identifi er is used as a mere reference for easier de- 
scription of a graph. In the lower compartment the attribute names together with their concrete values are depicted one per line. Figure 1 shows an attributed graph with two vertices and three data value nodes on the left-hand side, and the corresponding simplifi ed version of that graph on the right-hand side.
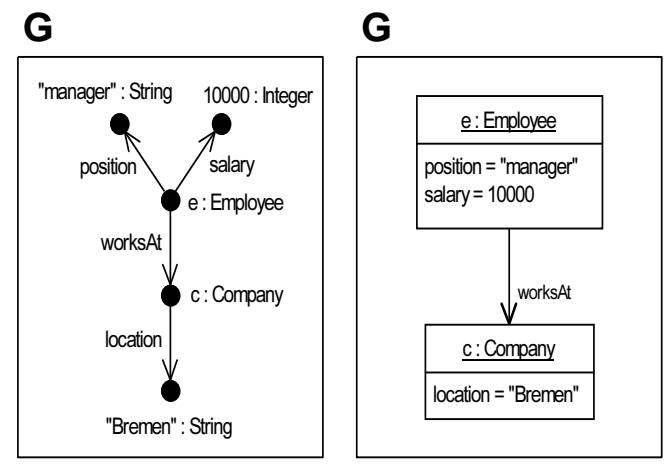

Figure 1. An attributed graph in two different notations

In this paper a graph transformation rule consists of three graphs $L, K, R$, called left-hand side, common part, and right-hand side, respectively. In order to increase the flexibility of rules, the data value nodes of the left-hand side are extended by a set $X$ of variables. Thus it is possible to specify variable values instead of concrete constant values for attributes in the left-hand side of a rule.

Attribute data value nodes of the right-hand side are somewhat more complex in order to allow for attribute value computation. We assume that every concrete data value is represented syntactically by a constant symbol. Let $B=\left(B_{i}\right)$ be the family of these constant symbols, indexed by the basic types listed above. Furthermore let $\Sigma_{B}(X)$ be the set of expressions over $B$ and $X$ which is defi ned as usual: an expression is either a constant $c \in B$, or a variable $x \in X$, or it is of the form $\omega\left(t_{1}, t_{2}, \ldots t_{n}\right)$ with $\omega$ being an operation symbol and the arguments $t_{i} \in \Sigma_{B}(X)$ being expressions of suitable types. In our case we use the usual operations, e.g. arithmetic operations on numbers, sign manipulation and the like. The data value nodes of the right-hand side are then all elements of $\Sigma_{B}(X)$. In fi gures the operations are written in infi $\mathrm{x}$ notation, i.e. $+(5, x)$ becomes $5+x$.

The two rule sides $L$ and $R$ are connected by a common part $K$, which is a subgraph of both $L$ and $R$. $K$ is a subgraph of $L$ if the nodes and edges of $K$ are subsets of the nodes and edges of $L$, respectively, and the nodes and edges coincide in their respective label, source and target mappings. Informally speaking a rule is applied to a given graph $G$ (called host graph) by fi nding a situation in $G$ that is specifi ed by $L$. Then the part corresponding to $L-K$ is deleted from $G$ and $R-K$ is glued to $G$. In fi gures depicting rules, the identifi er of vertices is used in order to indicate parts of the gluing graph $K$, i.e. a vertex with the same identifi er in the left-hand side and the right-hand side is an element that has to be preserved. Elements without identifi ers are either deleted from (in the left-hand side) or added to the graph (in the right-hand side).

In order to fi nd a situation specifi ed by $L$ in a host graph $G$, a so-called match 
is needed, i.e. a structure-preserving mapping (called graph morphism) of $L$ into $G$. A graph morphism maps the nodes and edges of one graph to the nodes and edges of the other graph such that labels and source and target nodes are preserved (ignoring isolated data value nodes). For our purposes we demand an injective match, i.e. equivalent images require equivalent preimages. Since no variable set is present in the host graph, variable value nodes from $X$ may be mapped to any data value node in the host graph, provided that the structural properties are preserved. Figure 2 shows a rule with a variable sal in the left-hand side and a match of $L$ in a host graph $G$. The match determines a variable binding for the variable data value nodes in $L$; for instance, $m(s a l)=10000$ in Figure 2. The same variable may be used more than once in the left-hand side via several edges leading to the corresponding variable data node. In fi gures, variables are printed in italics.

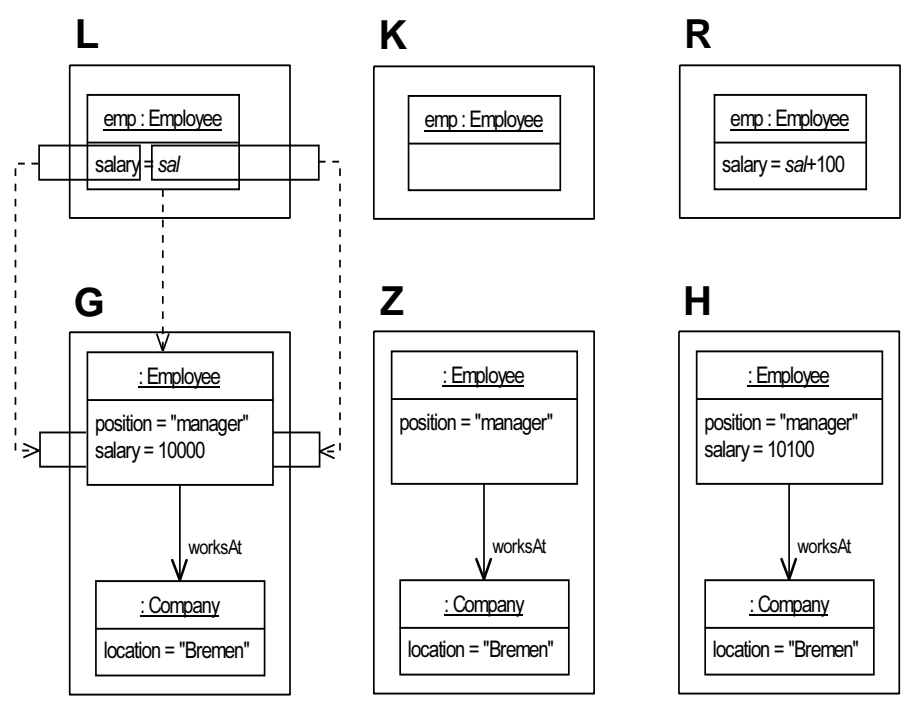

Figure 2. A rule $(L, K, R)$, a match $L \rightarrow G$ and the application to $G$

A rule $r=(L, K, R)$ is then applied to $G$ by deleting $m(L-K)$ from $G$. This is done by removing the vertices and the edges, together with possible dangling edges, i.e. edges with source or target in $m(L-K)$. This yields an intermediate graph $Z$. If $R$ contains visible data value nodes from $\Sigma_{B}(X)$, their expressions are evaluated using the variable binding $m$. If the expression is of the form $x$ with $x \in X$, the edge leading to $x$ is deleted and a new one with the same label leading to $m(x)$ is created. If the expression is of the form $\omega\left(t_{1}, t_{2}, \ldots, t_{n}\right)$, the operation and parameters are interpreted in the usual way (in case of $t_{i} \in X$ using $m\left(t_{i}\right)$ ) and the edge leading to that value node is replaced with an edge (again with the same label) leading to the result of the evaluated expression. This evaluation yields an instance $R^{\prime}$ of the right-hand side which has only visible data value nodes from the basic sets. Now $R^{\prime}-K$ is glued to $Z$. This is done by adding all vertices and edges from $R^{\prime}-K$ to $Z$, possibly gluing new edges to already present nodes, i.e. if $s(e) \in K$ for an edge $e$ of $R^{\prime}-K$, then $e$ is connected to $m(s(e))$ (and analogously for $t(e) \in K)$. Figure 2 shows the application of a rule $(L, K, R)$ by depicting $G$, the intermediate graph $Z$ and the result graph $H$ after successful rule application. 
The edge labeled salary is not part of $K$, thus it is deleted in $Z$ and added to $H$ as a new edge present in $R$ but not in $R^{\prime}-K$. The data value node 10000 becomes invisible in $Z$, and the data value node 10100 becomes visible in $H$.

Besides specifying a desired situation in the left-hand side of a rule, it is sometimes useful to specify a situation in the host graph that is not wanted. This is realized by a negative application condition (NAC). An NAC is a graph that extends parts of the left-hand side, i.e. a (possibly even empty) subgraph of $L$ is a subgraph of such a graph $N A C$. If the match of $L$ in the host graph $G$ can be extended to a match of $N A C$ in $G$, then the rule cannot be applied. Figure 3 shows the rule from Figure 2 together with an additional $N A C$ that prevents the rule from application if the salary to be increased is exactly 100000 .

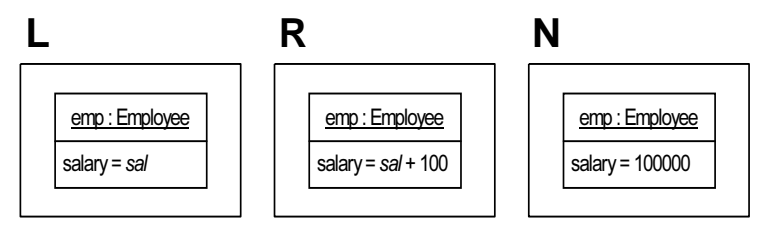

Figure 3. A graph transformation rule with an $N A C$

In our approach, we also employ a simpler form of the so-called transformation units [KK99,Kus00]. In our case a transformation unit comprises a set of local rules and a control condition. To apply a transformation unit to a given graph, the new graph is derived by applying the local rules according to the control condition. The semantics of the operators used inside control conditions will be explained where they occur in the following sections. We regard the application of a transformation unit as an atomic operation, similar to one rule application, since the intermediate graphs are not of any interest. For our purposes the transformation units are constructed in such a way that either their fi rst rule is not applicable or the whole unit can be applied successfully ${ }^{4}$. In our approach the semantics of control conditions differs from the original defi nition in that a failed application of a control condition yields the original (unchanged) graph. In the next section we provide an overview of the UML features that are covered by our approach supported by a very basic sample model.

\section{Covered UML Features}

We cover substantial aspects of the following UML features: Use case, class, object, statechart, and interaction diagrams (collaboration and sequence diagrams) and last but not least full OCL.

We support class diagrams for defi ning the structure, and interaction diagrams for realizing operations declared in the class diagram. An interaction diagram contains

4 A property like that would be hard to achieve for transformation units in general, but in the context of our work only a very small subset of all possible transformation unit constructions is needed. Due to this fact and the rule construction scheme in general, the mentioned property can be guaranteed. 
a sequence of messages calling either an operation of a class that in turn is realized by an interaction diagram or calling a predefi ned functionality like creating an object or setting an attribute value.

Use cases are likewise realized by interaction diagrams. A use case resp. its realization states which operations could be called by a user of the eventually implemented system and in which order this is done. Statechart diagrams specify the order in which operations on an object may be executed. The kind of statechart diagrams we support are so-called protocol machines, i.e., statechart diagrams with guards and events used as transition labels. Object diagrams are used to specify the system state to start the evolution with and to represent a part of the current state of the system.

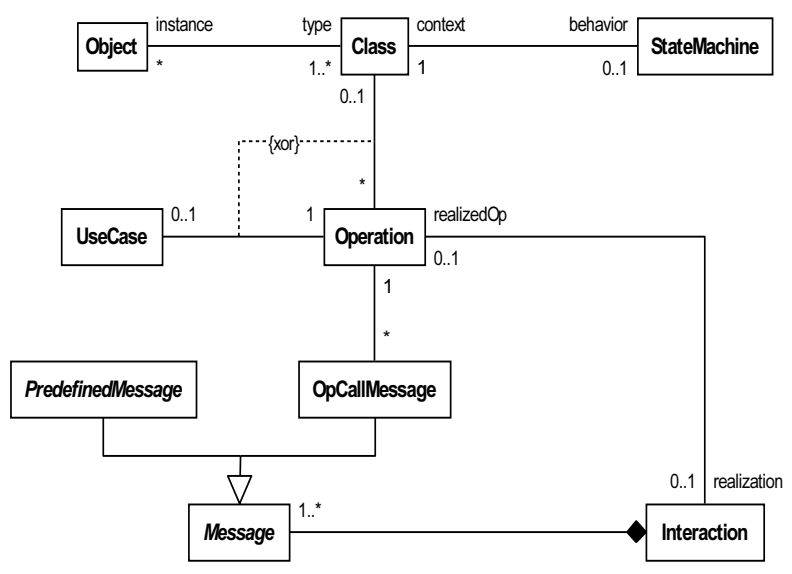

Figure 4. Connection between central modeling concepts

Fig. 4 gives an overview of the connections between the central concepts. We consider one class diagram and one use case diagram. This is no limitation because in our approach multiple class diagrams can be merged to one, and so can use case diagrams. Each class has zero or more operations. A use case is associated with exactly one operation that is not associated with a class. Each operation is realized by an interaction specifi ed in an interaction diagram. An interaction contains messages, which are either predefi ned (e.g. for creating an object or setting an attribute value) or which call an operation of a class. For each class there can be one state machine specifi ed in a statechart diagram. An object diagram instantiates the class diagram. We illustrate the usage and interplay of the diagrams by a representative excerpt of an example UML model of a drive-through restaurant.

The drive-through system consists of clients who enqueue themselves in the queue of a drive-through restaurant, submit orders, pay for meals, and eat. The restaurant produces meals and serves them to the clients.

The class diagram in Fig. 5 defi nes the structure of our example system. The class DriveThrough represents drive-through restaurants. A drive-through is visited by several clients who await being served. This is specifi ed by the association Visit between DriveThrough and Client. The two additional associations First and Last mark one client as the fi rst one in the queue and another client as the last one. 
The order in the queue is reflected by the association Queue. Clients can select an order by connecting to an Order object by the association Submit. The drivethrough is then expected to produce a Meal object corresponding to the order. This correspondence is managed by the attributes name resp. meal of the two classes. A meal that has been produced and is ready to be served is connected to the drivethrough by the association ToServe. A served meal is connected to a client by the association ToEat until it is eaten.

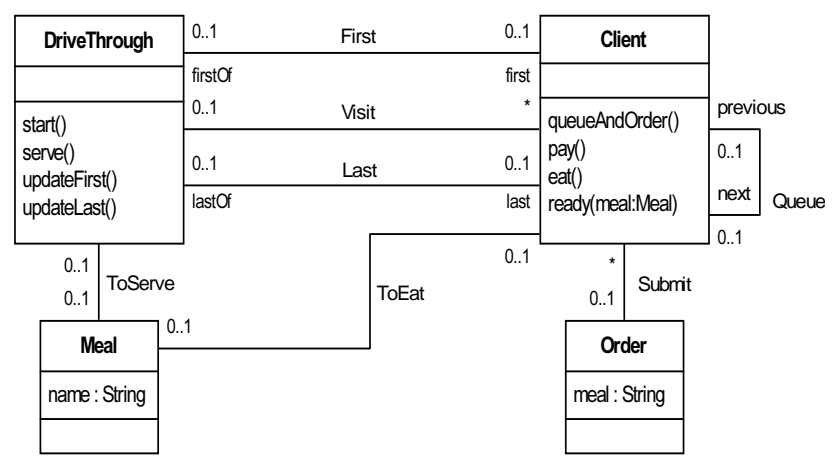

Figure 5. A class diagram

A use case diagram is used to show on a high level the possible interactions of a user with the eventually implemented system. A use case is a sequence of operations the user may call. The use case diagram only shows the names of the use cases; the corresponding sequence is specifi ed in an interaction diagram. In our example there is a use case startDriveThrough which enables the user to control the drivethrough, and a use case callClientToEat for "telling" an idle client to queue himself in the queue of a drive-through and submit an order. The user of the drive-through system is modeled as supervisor.

Before elaborating on the relatively low-level interaction diagrams, we take a look at statechart diagrams, which are used in a more high-level way in our approach. A statechart diagram specifi es the states an object of a certain class can be in, which operations may be executed in which state, and how the execution of an operation changes the state of the object the operation is running on. We assume that operations which do not occur in the statechart for the corresponding class are allowed to be executed in every state.

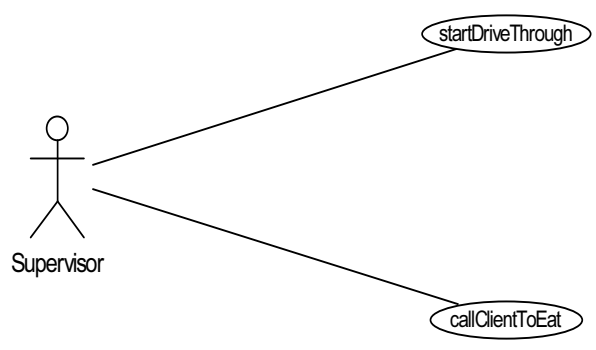

Figure 6. A use case diagram 
The statechart diagram in Fig. 7 specifi es the states a client can be in: idle, waiting and hasPaid. The initial state is the state idle, which means that once a client object is created it is in state idle. It is also specifi ed here that executing the operation queueAndOrder is allowed only in state idle and changes the state to waiting. The operation pay then changes the state to hasPaid and the operation eat then changes the state back to idle.

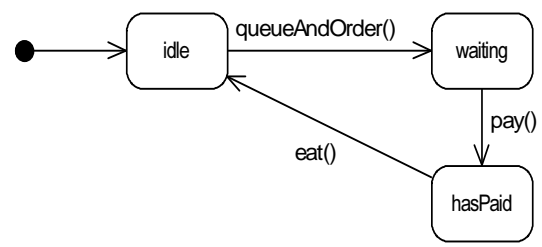

Figure 7. A statechart diagram for the class Client

As mentioned above, the operation sequences that constitute use cases are specifi ed in interaction diagrams. In this case we use collaboration diagrams but sequence diagrams could also be used, as explained below.

The collaboration diagram shown in Fig. 8 realizes the use case callClientToEat by specifying the messages the supervisor can send. In this case we have only one OpCallMessage as referred to in the metamodel in Fig. 4. The message (numbered with 1) is sent to a classifi er role representing a client and calls its operation queueAndOrder(). Other messages 2, 3 etc. could have followed for a larger use case. The sequence numbers at the beginning of the messages specify the order in which they are sent.

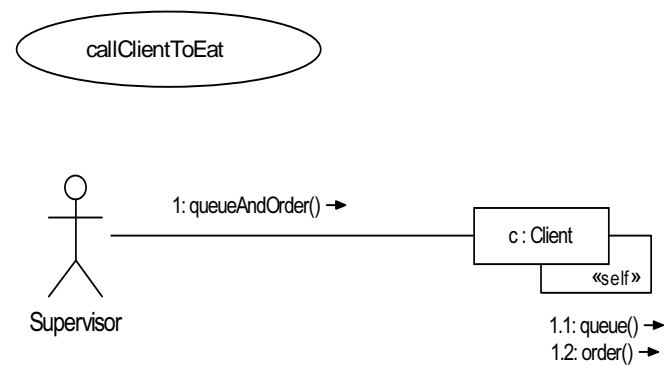

Figure 8. A collaboration diagram realizing the use case callClientToEat and the operation queueAndOrder of the class Client

The sequence numbers are nested in different depths to allow the realization of several operations in a single diagram. The present collaboration diagram also specifi es the operation queueAndOrder() on Client which is realized by fi rst calling queue() (sequence number 1.1) and then order() (sequence number 1.2) on the object that receives the message.

Fig. 9 shows a more complex collaboration diagram. The operation serve() is called by a supervisor on a drive-through object, which in turn calls several other operations by sending the messages 1.1 to 1.5 . Message 1.4 calls the operation ready(meal) on a client, which in turn is realized by the messages 1.4.1 and 1.4.2. 
The other messages call operations with a predefi ned effect like creating an object or setting an attribute value.

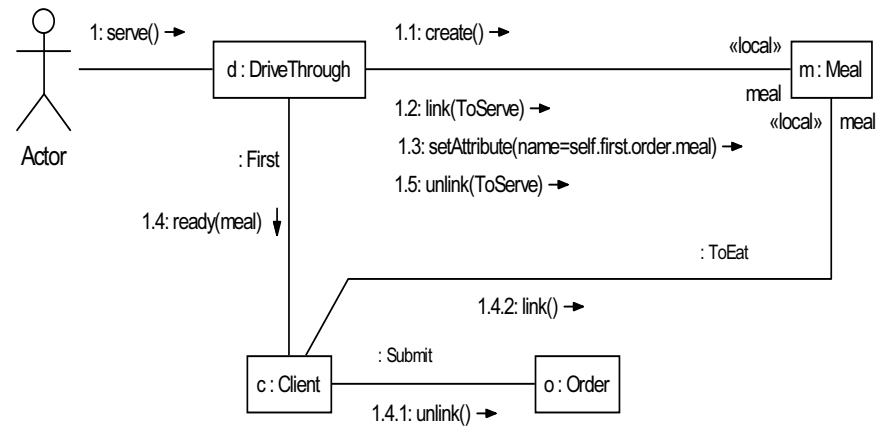

Figure 9. A collaboration diagram realizing the operations DriveThrough::serve() and Client::ready(meal:Meal)

The nodes in a collaboration diagram are called classifi er roles. They represent objects in the system state. The edges are association roles representing links. A message that is sent via an association role that is stereotyped with «local» means that the receiving classifi er role is stored in a local variable. The name of the variable is specifi ed by the role name. If the association has no stereotype, the interaction is underspecifi ed. In this case, the message is sent to an arbitrary object of the specifi ed class that is linked to the sender object by a link of the specifi ed association. A future version of the aforementioned tool will alert the modeler of this underspecifi cation and permit them to choose an actual receiver object.

The message 1.2: link(ToServe) specifi es that a link instantiating the association ToServe has to be created. Usually a link message needs parameters specifying the concrete objects that have to be linked as well as their association roles (especially if the arity of one association end is greater than one - in this case it is not always obvious which objects have to be linked). In the context of the given model it is not necessary to provide this information, since ToServe is specifi ed to associate one object of type DriveThrough with an object of type Meal. The actual classifi er roles to be linked are in this case the sender $d$ and the receiver $m$. In case of the link message 1.4.2: link() it is analogously determined which objects will be linked in what way. The usage of an instance of the association ToEat as a channel for the message in this case also determines that the link to be created will instantiate the association ToEat without explicitly providing it as a parameter.

Object diagrams are used in our approach to specify a part of an initial system state, that is the system state the modeler wants to start the system run with. The object diagram in Fig. 10 depicts such an initial system state for the drive-through example. There are one drive-through and four clients. Three of them are visiting the drive-through and have already ordered. The state of the objects is specifi ed by attached notes.

Having specifi ed the relevant parts of the system to be implemented, the modeler may execute the DriveThrough model to check for design flaws. Given the system state corresponding to the initial object diagram in Fig. 10, it could be checked 

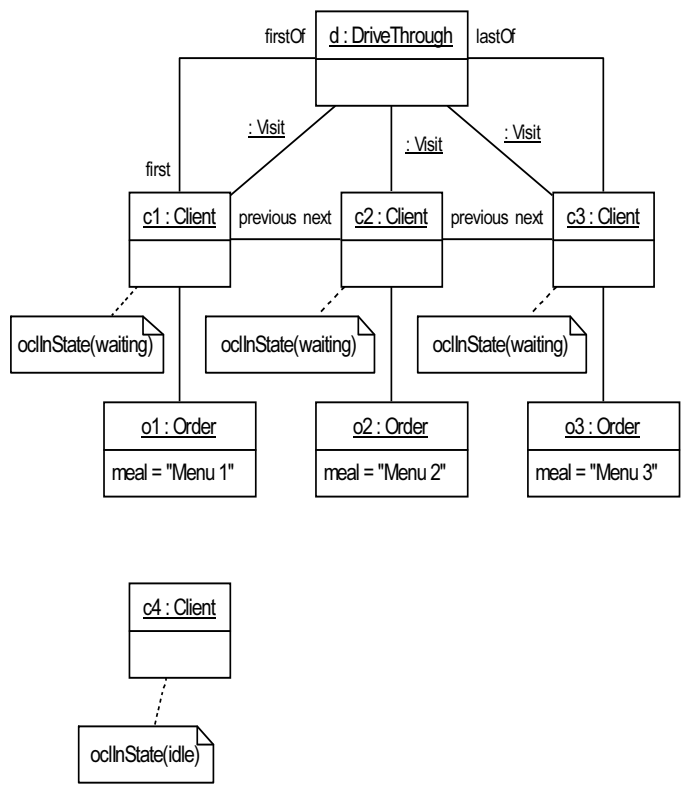

Figure 10. An object diagram specifying the initial system state

whether client $c 4$ can be inserted into the queue in the right position. It could also be checked whether $\mathrm{c} 4$ can be served, or whether the other three clients may submit further orders. A test could also reveal, whether a state is reachable, where all clients have been served according to their orders and the queue is empty. This would be a desired state, at least for the staff of the DriveThrough regarding closing time. An incomplete or contradictory specifi cation can also be revealed in the simulated system run. For instance, in case of a missing link a graph transformation rule relying on this link may never be applicable during the system run. If the modeler forgot to specify the link operation 1.4.2 in Fig. 9, the client would not be able to actually eat the ordered meal, and thus stay in the queue forever. By observing the simulation the modeler should realize this flaw.

In general our approach supports UML models comprising the following syntactical features:

- Use case diagrams with declaration of use cases

- Class diagrams with classes, $n$-ary associations, and inheritance

- Object diagrams with objects and links

- Statecharts with simple states labeled with guards and call events (protocol machines)

- Interaction diagrams with ordinary, «local», and «self» association roles, sequential, parallel, synchronous and asynchronous messages calling operations on objects or predefi ned operations

- OCL expressions in guards of statecharts and interaction diagrams and in arguments of messages

In our example, we only use synchronous messages, which are visualized by fi lled 
solid arrowheads. In contrast to this asynchronous messages are also supported. Before a message is sent it has to wait until the functionality invoked by the preceding synchronous message(s) has fi nished. Asynchronous predecessors do not have to be fi nished but they have to be sent.

OCL guards in square brackets in front of messages do not appear in this example but are also supported. Such an OCL condition has to be fulfilled to send the message.

Sequence and collaboration diagrams are based on the same information in the metamodel of UML 1.5 and thus are semantically equivalent (cf. [BRJ98], pages 249-250). It is even possible to convert one diagram type into the other without loss of information [CHK04]. However, in the concrete syntax of sequence diagrams the association roles are not visualized. If the association roles were nevertheless included in a sequence diagram, it could also be used instead of a collaboration diagram in the model.

In the next section we introduce the concept of a system state, which is the essential part of our approach.

\section{System States}

A system state is a snapshot of the system at some point of time during a system run. It contains attributed objects and links connecting them. So far this graph can be regarded as an object diagram. However, a system state contains two more important concepts: (1) object states, which are attached to objects according to the statechart diagrams, and (2) processes, which represent the actual execution of operations. Briefly, the main concepts of a system state are the following ones:

- Class vertices together with operation and association vertices represent the statical structure

- Inheritance is represented by connecting the relevant class vertices

- Object vertices represent existing instances of the connected class vertex

- Link vertices connected to object vertices represent instances of the connected associations

- Process vertices represent attached operations in execution

- State vertices represent the current state of an attached object

- Local variables are represented by local variable vertices

- State vertices attached to class vertices determine the initial state for new instances of that class

- State vertices attached to process vertices determine the state of the corresponding object after the execution of the process has fi nished

The abstract syntax of system states is shown in Fig. 11-13 by means of a metamodel. The part of the metamodel shown in Fig. 11 covers the basics of a system state, which are mainly concepts known from UML object diagrams: objects with 


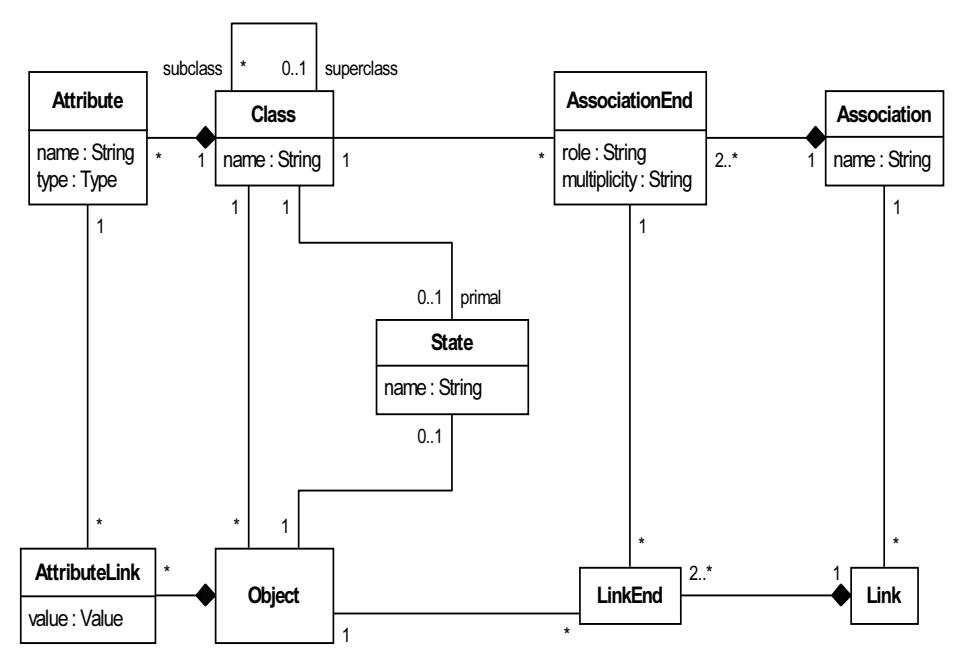

Figure 11. Abstract syntax of system states-the basics

attribute links and links together with link ends connecting the objects. These elements can be created or destroyed during a system run.

Additionally, the corresponding elements from the class diagram (classes, attributes, associations association ends) are also included and connected to their instances. These elements exist throughout a system run. An object, for instance, is connected to its class. Each attribute link is connected to an attribute and each object is connected to an attribute link for each attribute its class or one of its superclasses contains. Thus, attributes are inherited from superclasses to subclasses.

In addition to the common snapshot information, there are (object) states in a system state connected to objects. An object of a class, for which a statechart is given, can be connected to a state that has a name referring to a state from the statechart.

When an object is created during a system run there has to be a way to determine the initial state of the newly created object. This is accomplished by connecting the initial state (according to the statechart) to the class of the object.

\subsection{OCL in Graph Transformation}

As explained in the previous section a system state represents a current snapshot state of a system modeled by a given UML model. This UML model contains the object model $\mathcal{M}$ defi ned in [Ric02]. The object model defi nes all the available types as well as valid expressions, thus we use $\Sigma_{\mathcal{M}}$ (being the signature of $\mathcal{M}$ ) as the set of data value nodes in the following. For this reason vertices in rules are attributed with OCL values like 42 , Sequence $(1,2,3)$ or $o 2$ (being an object). A system state may only contain constant values, thus the OCL expressions in the rules have to be evaluated. This is achieved by employing $I\left(\Sigma_{\mathcal{M}}\right)$ as defi ned in [Ric02], which maps each type to a carrier set and each operation symbol to a function. The function for evaluating these expressions is not straightforward, as 


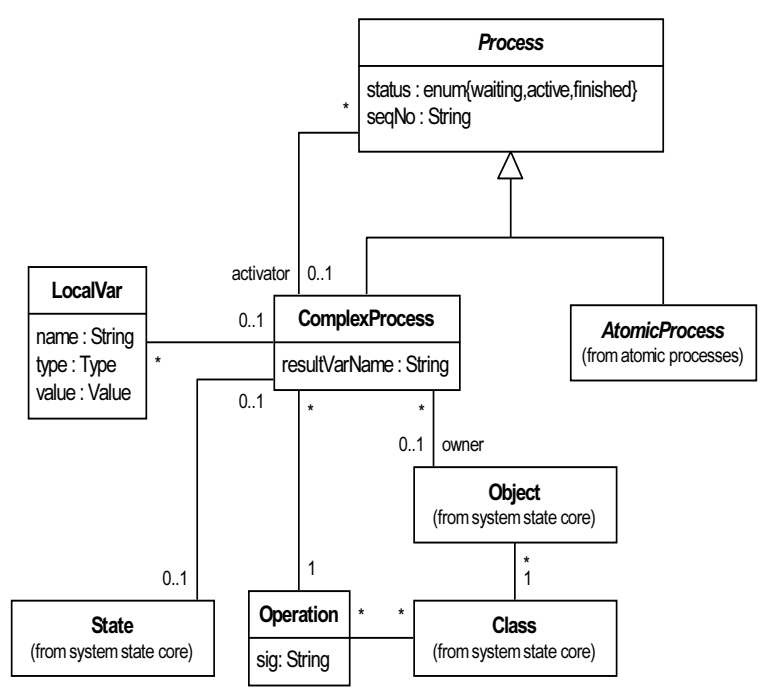

Figure 12. Kinds of processes

it depends not only on the variable assignment but also on a current system state, which in our case is represented by the host graph. The evaluation of an OCL term $e$ of type $t$ is defi ned in [Ric02] by a function $I[[e]]: E n v \rightarrow I(t)$, where Env is a set of environments $(\sigma, \beta)$ consisting of a state $\sigma$ of the system and a variable assignment $\beta$. We use this defi nition, where $\beta$ is determined by the match and $\sigma$ is derived from the current system state. This derivation is straightforward since the system state contains all the information included in a system state as defi ned in [Ric02] extended by current object states. Thus an ocllnState expression can be evaluated in our context as well.

The possibility of evaluating OCL expressions in the context of the current host graph yields a comfortable means of formulating further positive application conditions for a rule. An OCL application condition(AC) is a boolean OCL term, specifying a constraint on a match. A match $m$ satisfi es an AC $e$ if $I[[e]]$ evaluates to true in the context of the current host graph and the variable binding determined by $m$. So a rule with an AC may only be applied if a match is found that satisfi es the AC.

\subsection{Processes}

A very important concept of system states is that of a process. Roughly speaking, a process represents an operation in execution. As shown in Fig. 12 there are two different kinds of processes: complex processes representing user-defi ned operations in execution and atomic processes representing predefi ned operations in execution. A user-defi ned operation is an operation declared in a class diagram and specifi ed by an interaction diagram or a use case, likewise specifi ed by an interaction diagram. In the fi rst case, a corresponding process is executed on an owner object, 
which is an object of the class the operation is defi ned for. A complex process can activate other processes, both complex and atomic, and it can have local variables, i.e. variables only visible in the scope of the process. All processes have a sequence number and a status. The sequence number (seqNo) refers to the message in an interaction diagram that corresponds to the process. The status can be waiting, active or finished. A waiting process represents a called operation that has not been started yet. A fi nished process often is a precondition for calling another operation. A process can also be connected to a state. This is necessary to determine the state of the owner object once the process is fi nished. Upon termination of that process, the object will be connected to that state.

Fig. 12 also shows that operations are not only connected to one class but possibly to many. An operation is connected to the class for which it is declared in the class diagram and also to all its subclasses that inherit the operation. An operation is not connected to the subclasses that override the operation by having an operation with the same signature. These connections are needed to ensure that the correct operation is executed on an object that inherits or overrides an operation.

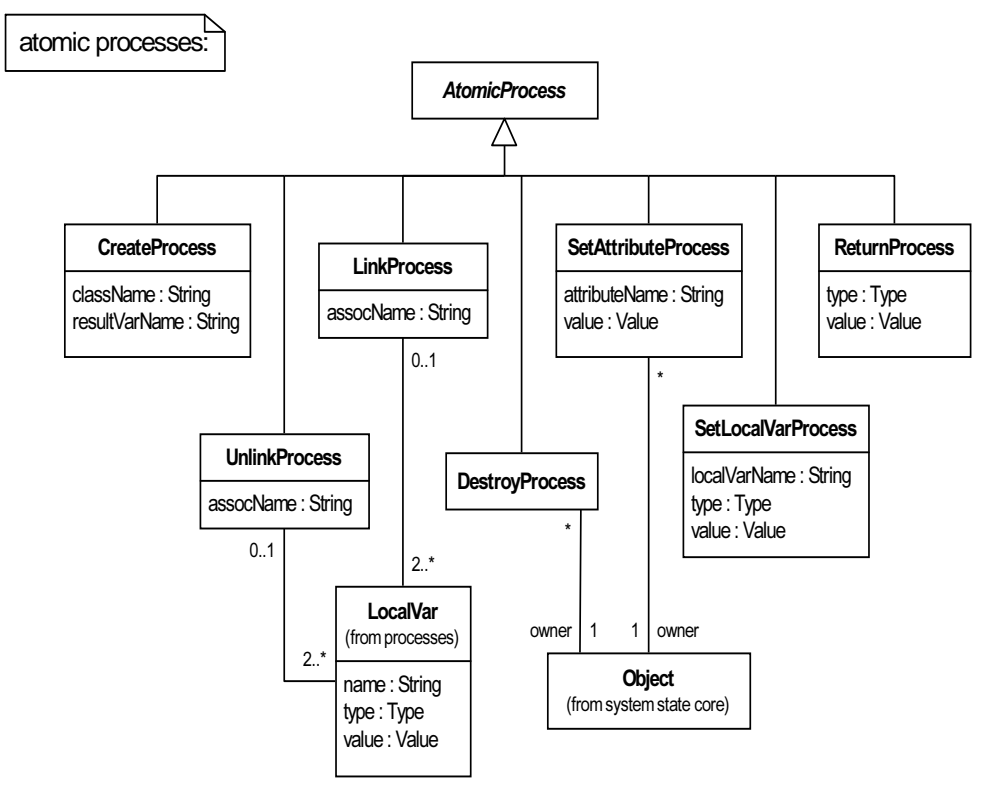

Figure 13. Atomic processes

Fig. 13 shows the atomic processes corresponding to the predefi ned messages mentioned earlier. They are called atomic because they do not activate other processes. We need seven different kinds of atomic processes that handle the low-level modifi cation of the system state:

CreateProcess A create process creates a new object of the class given by className. The new object is returned to the activator process by setting its local variable given by resultVarName.

DestroyProcess A destroy process removes its owner object from the system state. LinkProcess A link process establishes a link between objects. The association to 
be used is given in assocName and the objects to be linked are given by a set of local variables. Each variable has an association role as name and an object of appropriate type as value.

UnlinkProcess An unlink process removes a link between objects. The local variables are analogous to the variables of a link process.

SetAttributeProcess This kind of process sets the attribute given by attributeName of its owner object to a given value.

SetLocalVarProcess This kind of process sets the local variable given by attributeName of its activator process to a given value. If the variable does not exist yet it is created.

ReturnProcess A return process fi nishes its activator process by returning a value to it.

In the following section, we explain how an integrated UML specifi cation is translated into a graph transformation system, i.e., how the initial system state and the rules changing the system state are constructed.

\section{Translation into a Graph Transformation System}

In this section we present an informal overview of the translation of the given UML model into a graph transformation system. In general, the graph transformation system corresponding to the given UML model is built in the following way:

- construct the initial system state using information of the use case, class, object, and statechart diagrams

- create a rule for every use case

- generate a transformation unit for every method specifi ed in an interaction diagram

- add the predefi ned rules and transformation units to the graph transformation system

In the next section we will describe how the initial system state is constructed. The second section deals with the generation of rules resp. transformation units that depend on the model. The set of predefi ned rules, which do not depend on the model, are introduced in the third section. Finally a brief outline of the fundamental design of the prototypic implementation is presented.

\subsection{Initial System State}

The initial system state contains structural information about the system at the time the program starts. The construction of the initial system state considers the use case-, class-, object-, and statechart diagram supplied by the modeler.

For each use case there is an operation in the system state. It also contains all classes, operations, attributes, associations and association links from the class diagram. The initial state in the statechart diagram for a class is connected to this 
class. Operations declared in a class are connected to this class and the subclasses that do not override them.

The objects from the object diagram are added together with their links and attribute links. The objects are attached to states according to the notes from the object diagram or (in case there is no note at an object with an associated statechart diagram) to the initial state of the corresponding statechart diagram.

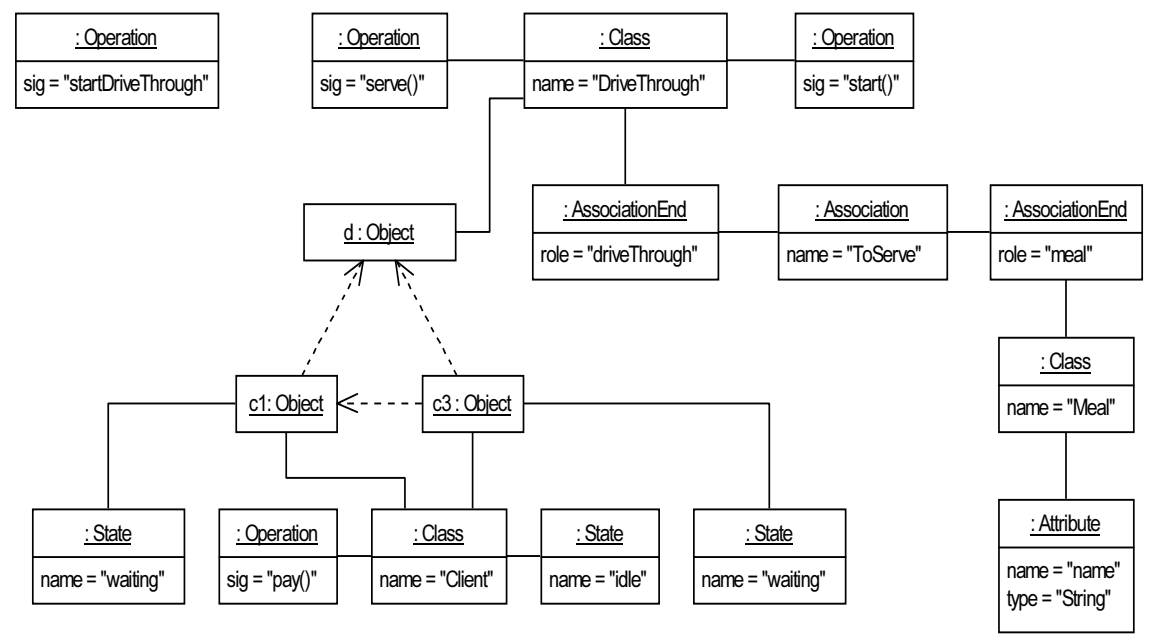

Figure 14. Start system state for the drive-through example

Fig. 14 shows a part of the initial system state of the drive-through example as object diagram instantiating the system state metamodel. Typically, system states in this notation are very large and diffi cult to handle for human beings. Therefore, a tool like the one presented in Sect. 6.5 would depict a system state in a more comprehensible way, e.g. by hiding class, attribute and operation vertices as it is usually done with object diagrams. The graph transformation rules however work on this complex structure.

Due to the complexity of the graph, Fig. 14 shows only an excerpt of it. In the upper left, you can see an Operation vertex that represents the use case startDriveThrough. The objects $d, c 1$ and c3 represent a drive-through resp. two clients (the other two clients are not shown). The dashed arrows just indicate the relations of the objects, abstracting from the links and link ends that actually constitute these relations.

During a system run, the system state is modifi ed by graph transformation rules. Basically we need two kinds of rules: Rules that depend on the given model and rules that do not, i.e., predefi ned rules. The following two subsections discuss these rules and how to construct them.

\subsection{Rules Depending on the Model}

The initial system state does not contain any processes, i.e., there is no operation that is called and waiting to be executed. This is what the use cases are needed for: For every use case we construct a rule that adds a ComplexProcess vertex with 
local variables for holding the arguments where necessary.

Fig. 15 shows the rule for the use case startDriveThrough. The rule creates a new ComplexProcess connected to the Operation with the name startDriveThrough. The status is set to waiting and the sequence number is set to 0 (because this process is not created by a message from an interaction diagram).

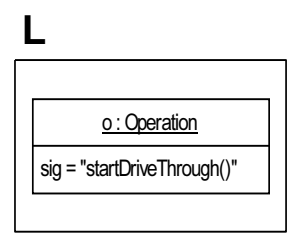

\section{$\mathbf{R}$}

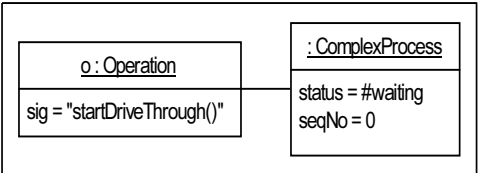

Figure 15. Rule for creating a use case process

With this kind of rule, we are now able to add processes to the system state in order to actually start a system run. Next we need rules that handle these processes, i.e., change the system state according to the semantics specifi ed in the interaction diagrams. For every operation specifi ed by an interaction diagram, we construct a set of rules. This is done for every operation no matter whether it belongs to a class or to a use case.
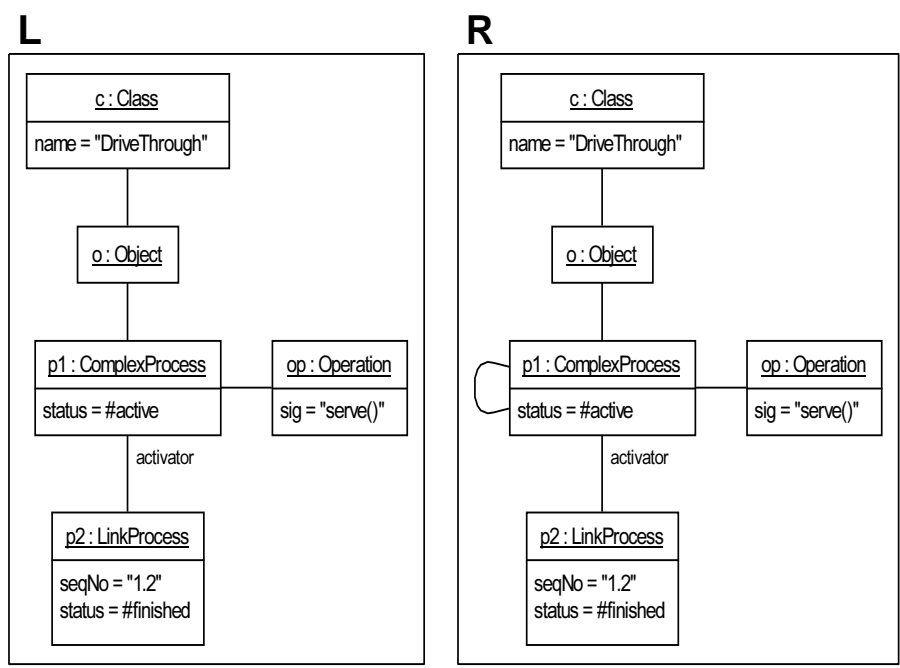

Figure 16. Sending a message: step 1

A user-defi ned operation calls several other operations. An interaction diagram specifi es which other operations are called and in which order this has to be done. So an interaction diagram contains messages that are sent between classifi er roles in a specifi c order. Each message represents the call of either a user-defi ned operation (of a class) or it represents the call of a predefi ned operation (like setting an attribute value). Every sent message corresponds to the creation of a new process vertex.

As an example we discuss the transformation unit that is necessary to handle the sending of the message 1.3 of the interaction diagram for DriveThrough::serve() as depicted in Fig. 9. 
The message 1.3 is sent during the execution of the operation 1:serve of class DriveThrough. Therefore the new process vertex should only be created if there actually is a process ( $\mathrm{p} 1$ ) of this operation (op) running on an object (o) of the desired class (c). This process represents the activator message of the message that corresponds to the process vertex to be created. This activator process vertex is marked with a loop for further rule application. Since in this case the regarded message is not the fi rst one sent by the activator message, the status of the activator process vertex has to be \#active. Furthermore the execution of the operation 1.2 should have been fi nished, thus the status of the corresponding process vertex (p2)must be finished. The fi rst rule 11 depicted in Figure 16 does exactly that.
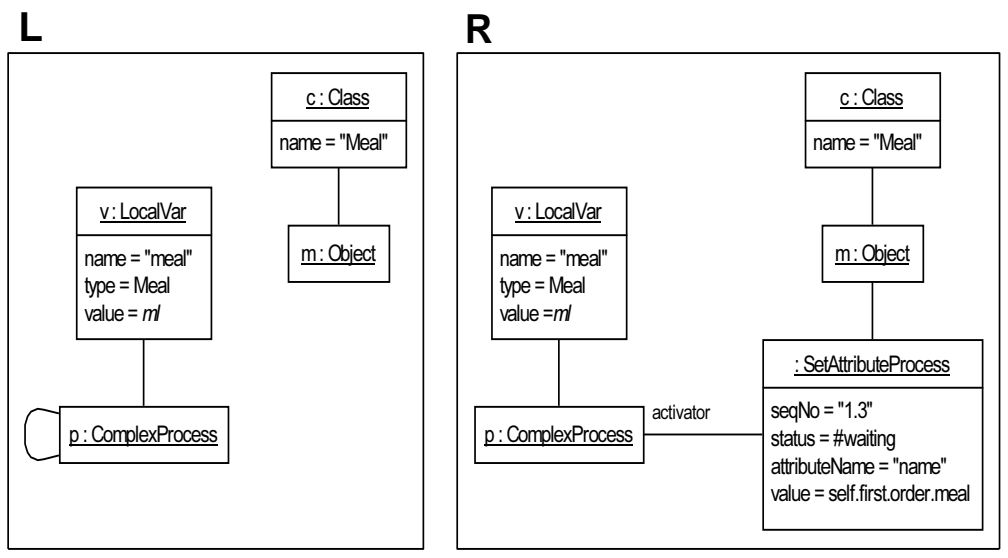

Figure 17. Sending a message: step 2

The second rule $\mathrm{r} 2$ creates a waiting process. This can be regarded as actually sending the message. The considered message in our example is sent to an object stored in a local variable meal as indicated by the stereotype «local» and the role name meal. In order to attach the new waiting process vertex to the correct object vertex, the left-hand side of the rule demands the presence of the LocalVar vertex attached to the activator process vertex. This LocalVar vertex has the value Meal which refers to the Object vertex connected to the class vertex with the name Meal. The new process vertex with status \#waiting will be connected to that object vertex and its activator process vertex. Its other attributes are set according to the collaboration diagram. In particular, the value is set to an OCL expression that is evaluated while applying the rule. In addition, the rule removes the loop from the activator process. The two rules have to be applied one after the other, thus the control condition of the transformation unit is $r 1 ; r 2$, meaning that fi rst the rule $r 1$ and after it rule $\mathrm{r} 2$ has to be applied.

In general, there are a lot of circumstances to consider when constructing such a transformation unit for sending a message. After having shown the concrete example, we will only outline some details not covered by the example.

- When a complex process is created instead of an atomic one, it has to be connected to the correct Operation vertex. This is the reason why two rules are needed in general, otherwise there would be two Operation vertices in one rule 
that possibly represent the same operation. Because we use injective matching, this would be impossible.

- Asynchronous predecessors are represented by processes with no specifi ed status in the rule, i.e., an asynchronous predecessor does not have to be fi nished.

- Parallel messages are sent by creating corresponding processes at the same time in one rule.

- If the message to be sent is the message that starts the execution of a waiting process and this execution is allowed only in certain states according to a statechart diagram, then the rule must only be applicable with a match containing a receiver object in the correct state. The rule then disconnects the state from the object, which is then "between" two states, and connects the next state to the new process. The object obtains its new state by another rule when the process has fi nished.

- If a message is sent via an association role without stereotype, a process is created and connected to an arbitrary object (of the specifi ed class) that is linked to the sending object (by a link of the specifi ed association). Thus, the rule includes links, associations, link ends etc. In addition, the rule creates a new local variable that stores the chosen object, in case other messages are sent via the same association role.
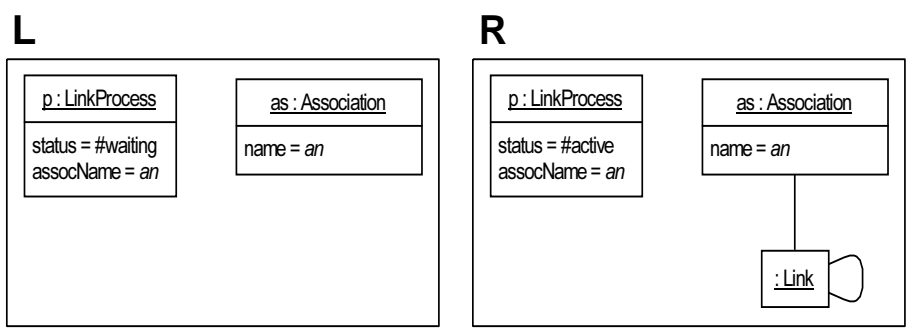

Figure 18. Rule createLink

\subsection{Predefined Rules}

Predefi ned messages do not call a user-defi ned operation but rather a predefi ned operation. There are messages for creating an object of a specifi c class, destroying an object, connecting objects with a link of a given association, unlinking objects, setting an attribute value, setting a local variable value, and returning a result. Corresponding to these messages there are atomic processes that are not associated with an operation but instead with other information needed for the task. These atomic processes have already been shown in Fig. 13. The rules that handle these processes are also predefi ned, i.e., they are independent from the user model.

There is a predefi ned rule or even transformation unit for each kind of atomic process. In addition to these, there are rules for collecting garbage. These rules remove fi nished processes that are no longer needed as preconditions for other processes, and local variables that are no longer attached to a process vertex. These rules are 


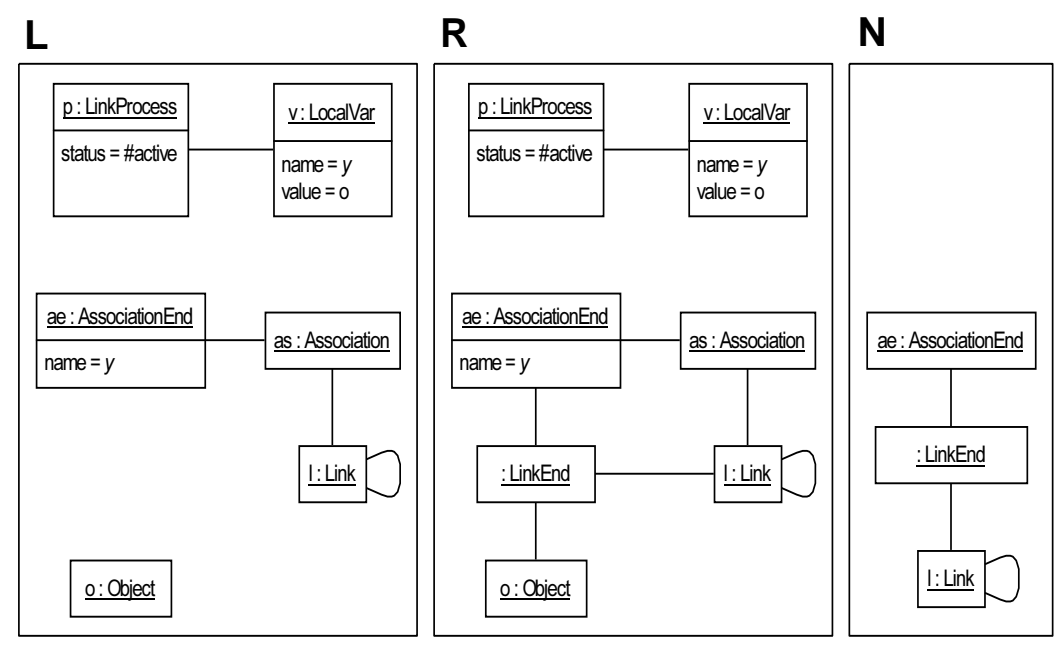

Figure 19. Rule createLinkEnds

applicable whenever such garbage exists in the system state.

In the following, we will present the transformation unit for handling a link process in more detail.

A LinkProcess has several local variables, each of them indicating the object that is supposed to play a certain role in the link that shall be created. The following transformation unit manages to create and connect a Link vertex and LinkEnd vertices that connect the objects as requested.

\section{link}

rules: createLink (Fig. 18)

createLinkEnds (Fig. 19)

finishLinking (Fig. 20)

cond: createLink; createLinkEnds!; finishLinking

To execute a LinkProcess, the rule createLink is fi rst applied to create a Link vertex with a flag. This new vertex is connected to the association indicated by assocName. This rule also changes the status of the process to \#active. Then, the rule createLinkEnds is applied as long as possible to create LinkEnd vertices for all objects that are to be linked and to connect these vertices. The NAC ensures that only one link end is created per association end. This rule is supposed to attach new LinkEnd vertices only to the Link vertex that was created earlier in the application of this transformation unit, which is ensured by the flag. The flag is removed and the process is fi nished by the rule finishLinking.

In general the use of transformation units is employed in order to encapsulate functionality into transactions. This is due to the fact that certain rules cannot change the system state in one step. Since the intermediate graphs are of no interest (especially since the graph transformation background of the actual system state run is hidden from the modeler) they are thus neither visible nor accessible to the mod- 


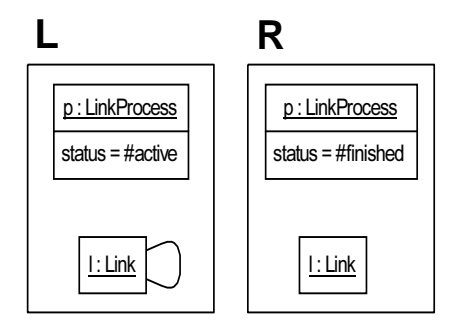

Figure 20. Rule fi nishLinking

eler. The information from the model is not reflected in the structure of the control conditions. Therefore information from the model, (e.g. the order of message calls) is still needed in the rules (e.g. sequence number).

\subsection{Inheritance}

In a system state an object has attribute values that are stored in AttributeLink vertices for all attributes that are declared in its class as well as in classes it inherits. The creation of the initial system ensures this, and the transformation unit that handles the creation of a new object also respects this fact. Therefore, a class inherits the attributes of its superclasses. Associations are implicitly inherited by the construction of the transformation unit that realizes a Link operation (for more details cf. [Zie05]). An important property of object-oriented systems that is supported by our approach is subtype polymorphism. Here an operation may be performed differently in different classes all of which have a common superclass with said operation. In the context of our approach this happens whenever a message is sent to a classifi er role associated with a class that has subclasses which override the called operation. In this case either the operation of the superclass or one of the overriding operations is called, depending on the class of the object that actually receives the message. We only allow to override an operation with another operation that has exactly the same signature (called invariant overriding (cf. e.g. [AC98]) in order to avoid typing problems. Such an overridden method has to be specifi ed in its own interaction diagram.

This is possible, since an Operation vertex is not only connected to the one class that declares it, but also to all of its subclasses that do not override it.

\subsection{Implementation}

Currently a prototype for the concepts discussed in this paper is being implemented. The goal of this prototype is to visualize the evolution of the system state. When provided with a model, the prototype automatically generates the graph transformation rules and the initial system state graph. A graphical user interface then permits the user to view the evolution of the system state step by step and to examine the current state by querying it using OCL.

For this reason the prototype must be able to perform graph transformations as well as to evaluate OCL expressions. Instead of implementing a new tool for these pur- 
poses, we chose to combine two well established tools. The graph transformation part is done by AGG [AGG05] and the evaluation of OCL expressions is performed by the USE tool [USE05]. An obvious choice for the tool implementation would have been the Fujaba tool [FNTZ98], which already couples graph transformation and UML. But since the UML part has to be handled by the USE tool anyway (in order to evaluate OCL expressions), any graph transformation engine capable of applying the rules generated by our approach suffi ces. Since the developers of the prototype are very familiar with AGG and its API, it has been the preferred choice as underlying graph transformation engine.

The prototype (hence UGT-UML to Graph Transformation) reads a USE specifi cation of a UML model that is compatible with our approach. It then generates the set of graph transformation rules according to the ideas presented in this paper. Additionally, the initial host graph is constructed from the object diagram the modeler provides. The GUI of UGT then displays this initial graph and the use case names as specifi ed in the model. The user may now select a use case to be executed. In this case, the rule that starts the execution of this use case is applied to the host graph. Now the user may click the step button and thus derive a next step in the system state evolution. Internally UGT calculates the next step by randomly choosing one of the rules that are applicable and letting AGG apply it. This may be done until no further rule is applicable. If this is the case, the use case is completely fi nished. As well as letting the system decide upon the next step, the user may control the flow of execution. Imagine a state with two processes in status waiting or active that can both be executed in the next step. In this case the user may decide what process is executed next by simply double-clicking it. If the process is specifi ed to expect parameters, the user is prompted with an input fi eld and forced to provide the necessary parameters. Figure 21 shows a screenshot of UGT in action. A system state is displayed with three clients visiting a drive-through, each of them with a submitted order. The user chose the use case startDriveThrough to be started, which resulted in the corresponding process being added to the system state.

By executing the system state step by step, the user can gain insight into the modeled system. Furthermore, UGT allows the evaluation of OCL expressions at any step in the system state. The OCL evaluation window displaying an OCL query and its evaluated result can be seen in the lower part of fi gure 21. Due to this feature, it is possible to check whether invariants hold during the execution of operations or even complete use cases. Note that UGT completely hides the graph transformation basis of this approach from the user. They do not need to know about the rule generation, or the fact that graph transformation is used to derive the next step of the system state.

\section{Conclusion and Future Work}

We have presented a conceptual approach for defi ning a semantics for UML based on the translation of a given UML model into a graph transformation system. To demonstrate our approach an example model comprising several UML diagrams 


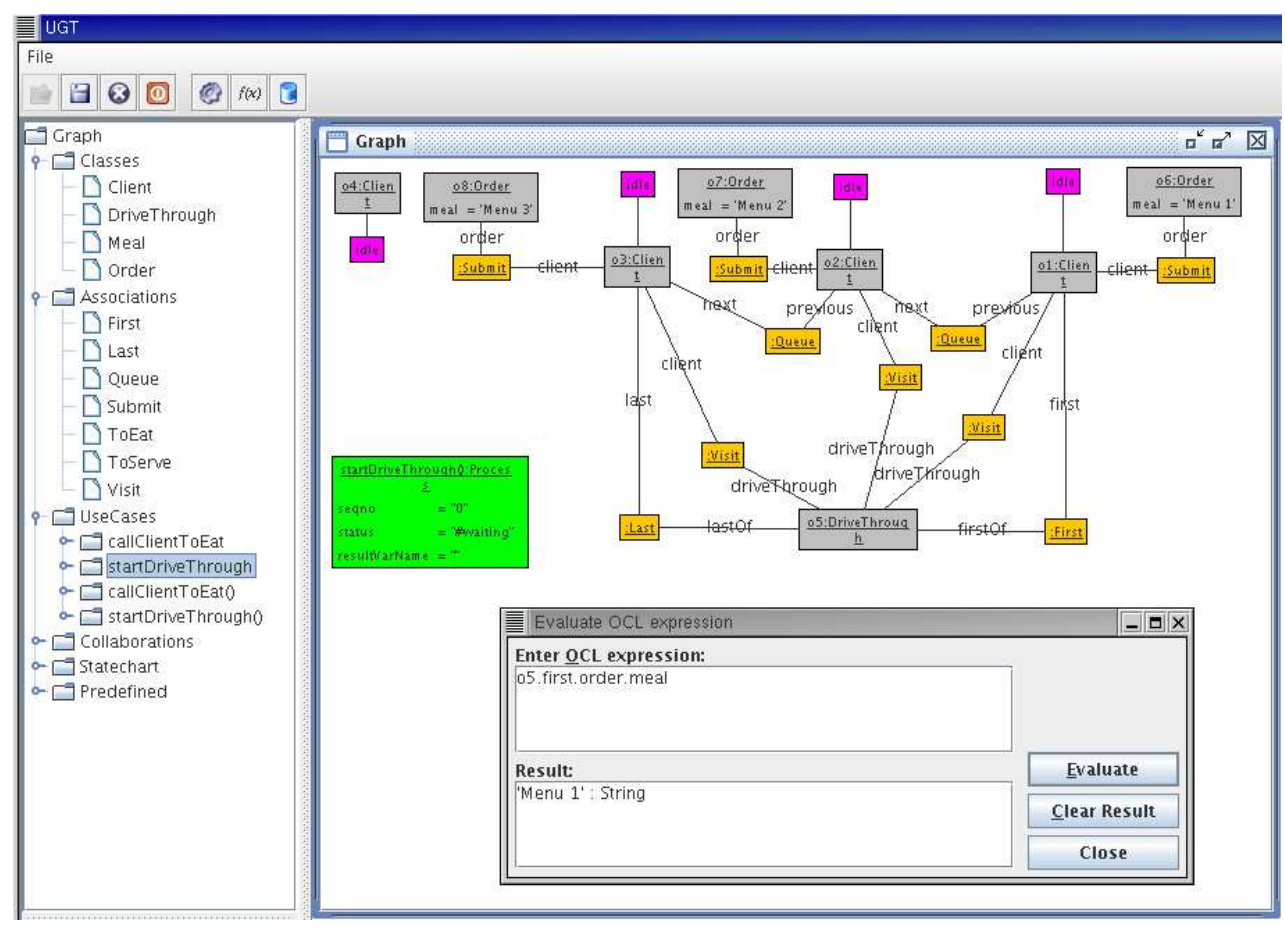

Figure 21. Screenshot of UGT

has been introduced. Next we have described our idea of a system state by means of a metamodel followed by a discussion of the translation of a given model into model-depending and predefi ned graph transformation rules by example. Finally the basic concepts of the prototypic software implementing this approach have been addressed. The prototype translates a given UML model into a graph transformation system and allows to monitor the evolution of the system state step by step.

The next goal is to complete the prototype implementation and to further enhance its GUI. As the approach and the tool are suitable for early stages of the software development process, it might become impractical when using large and very detailed models. In this case the aforementioned GUI should allow the user to choose different views on the system run, like e.g. hiding objects and their details that are of no interest in a certain situation.

An interesting topic would be the integration of further diagram types like activity diagrams into our approach. We will also investigate whether and how the diagrams already covered can be extended with yet missing UML features. These include composite states and concurrent ones in statechart diagrams and «include $\gg$ and $\ll$ extend $\gg$ relationships between use cases.

It may also be worth investigating whether a set of elementary templates can be provided for the rules that depend on the model. Currently every rule has to be generated from scratch for every model. Rule templates would provide a better maintainability in the sense that central concepts could be changed in one place instead of different rules.

Case studies will provide feedback on the practicability of the approach and tool. In 
particular, more insight is needed into the process of asserting properties of UML models on the basis of our approach, for instance, based on transformation invariants. In this way our approach will automatically benefi $t$ from future results in the fi eld of graph transformation.

\section{References}

[AC98] Martín Abadi and Luca Cardelli. A Theory of Objects. Springer, 1998.

[AGG05] The Attributed Graph Grammar System AGG, last revision 2005. http://tfs.cs.tuberlin.de/agg.

[And98] Andy Schürr and Andreas J. Winter. UML Packages for PROgrammed Graph REwriting Systems. In Hartmut Ehrig and Gregor Engels and Hans-Jörg Kreowski and Grzegorz Rozenberg, editor, TAGT, volume 1764 of Lecture Notes in Computer Science, pages 396-409. Springer, 1998.

[BCI03] Boldsoft, Rational Software Corporation, and IONA. Response to the UML 2.0 OCL RfP (ad/2000-09-03), January 2003. http://www.klasse.nl/ocl/oclsubm.html.

[BCR00a] Egon Börger, Alessandra Cavarra, and Elvinia Riccobene. An ASM Semantics for UML Activity Diagrams. In T. Rus, editor, Proc. Int. Conf Algebraic Methodology and Software Technology (AMAST'2000), volume 1816 of LNCS, pages 293-308. Springer, 2000.

[BCR00b] Egon Börger, Alessandra Cavarra, and Elvinia Riccobene. Modeling the Dynamics of UML State Machines. In Yuri Gurevich, Philipp W. Kutter, Martin Odersky, and Lothar Thiele, editors, Abstract State Machines, volume 1912 of LNCS, pages 223-241. Springer, 2000.

$\left[\mathrm{BHH}^{+}\right.$97] Ruth Breu, Ursula Hinkel, Christoph Hofmann, Cornel Klein, Barbara Paech, Bernhard Rumpe, and Veronika Thurner. Towards a formalization of the Unified Modeling Language. In Mehmet Aksit and Satoshi Matsuoka, editors, ECOOP'97 - Object-Oriented Programming, 11th European Conference, volume 1241 of LNCS, pages 344-366. Springer, 1997.

[BRJ98] Grady Booch, Jim Rumbaugh, and Ivar Jacobson. The Unifi ed Modeling Language User Guide. Addison-Wesley, 1998.

[CEK01] Tony Clark, Andy Evans, and Stuart Kent. The metamodelling language calculus: Foundation semantics for UML. In Heinrich Hussmann, editor, Fundamental Approaches to Software Engineering, 4th International Conference, FASE 2001, held as Part of the Joint European Conferences on Theory and Practice of Software, ETAPS 2001, Genova, Italy, April 2-6, 2001, Proceedings, volume 2029 of LNCS, pages 17-31. Springer, 2001.

[CF04] Alessandra Cavarra and Juliana Küster Filipe. Formalizing Liveness-Enriched Sequence Diagrams Using ASMs. In Wolf Zimmermann and Bernhard Thalheim, editors, Abstract State Machines, volume 3052 of LNCS, pages 6277. Springer, 2004. 
[CHK04] Björn Cordes, Karsten Hölscher, and Hans-Jörg Kreowski. UML interaction diagrams: Correct translation of sequence diagrams into collaboration diagrams. In M. Nagl, J. Pfaltz, and B. Böhlen, editors, AGTIVE'03 Proceedings, Lecture Notes in Computer Science, 2004. To appear.

[CRS03] Alessandra Cavarra, Elvinia Riccobene, and Patrizia Scandurra. Integrating UML Static and Dynamic Views and Formalizing the Interaction Mechanism of UML State Machines. In Egon Börger, Angelo Gargantini, and Elvinia Riccobene, editors, Abstract State Machines, volume 2589 of LNCS, pages 229243. Springer, 2003.

[EEKR99] Hartmut Ehrig, Gregor Engels, Hans-Jörg Kreowski, and Grzegorz Rozenberg, editors. Handbook of Graph Grammars and Computing by Graph Transformation, Vol. 2: Applications, Languages and Tools. World Scientific, Singapore, 1999.

[EHKG02] Gregor Engels, Reiko Heckel, Jochen Malte Küster, and Luuk Groenewegen. Consistency-preserving model evolution through transformations. In Jean-Marc Jézéquel, Heinrich Hussmann, and Stephen Cook, editors, UML 2002 - The Unifi ed Modeling Language. Model Engineering, Languages, Concepts, and Tools. 5th International Conference, Dresden, Germany, September/October 2002, Proceedings, volume 2460 of LNCS, pages 212-226. Springer, 2002.

[EK99] Andy Evans and Stuart Kent. Core meta-modelling semantics of UML: The pUML approach. In Robert France and Bernhard Rumpe, editors, UML'99 The Unifi ed Modeling Language. Beyond the Standard. Second International Conference, Fort Collins, CO, USA, October 28-30. 1999, Proceedings, volume 1723 of $L N C S$, pages 140-155. Springer, 1999.

[EKMR99] Hartmut Ehrig, Hans-Jörg Kreowski, Ugo Montanari, and Grzegorz Rozenberg, editors. Handbook of Graph Grammars and Computing by Graph Transformation, Vol. 3: Concurrency, Parallelism, and Distribution. World Scientific, Singapore, 1999.

[EW01] Rik Eshuis and Roel Wieringa. A real-time execution semantics for UML activity diagrams. In Heinrich Hussmann, editor, Fundamental Approaches to Software Engineering, 4th International Conference, FASE 2001, held as Part of the Joint European Conferences on Theory and Practice of Software, ETAPS 2001, Genova, Italy, April 2-6, 2001, Proceedings, volume 2029 of LNCS, pages 76-90. Springer, 2001.

[FELR98] Robert B. France, Andy Evans, Kevin Lano, and Bernhard Rumpe. The UML as a formal modeling notation. Computer Standards \& Interfaces, 19(7):325-334, November 1998.

[FM04] Stephan Flake and Wolfgang Mueller. An ASM Definition of the Dynamic OCL 2.0 Semantics. In Thomas Baar, Alfred Strohmeier, Ana Moreira, and Stephen J. Mellor, editors, Proc. Int. Conf Unifi ed Modeling Language (UML'2004), volume 3273 of LNCS, pages 226-240. Springer, 2004. 
[FNTZ98] T. Fischer, J. Niere, L. Torunski, and Albert Zündorf. Story diagrams: A new graph transformation language based on UML and Java. In H. Ehrig, G. Engels, H.-J. Kreowski, and G. Rozenberg, editors, Proc. Theory and Application to Graph Transformations (TAGT'98), Paderborn, November, 1998, volume 1764 of LNCS. Springer, 1998.

[GZK03] Martin Gogolla, Paul Ziemann, and Sabine Kuske. Towards an integrated graph based semantics for UML. In Graph Transformation and Visual Modeling Techniques (GT-VMT 2002), volume 72 of ENTCS, 2003.

[HKT02] Reiko Heckel, Jochen Malte Küster, and Gabriele Taentzer. Confluence of typed attributed graph transformation systems. In Andrea Corradini, Hartmut Ehrig, Hans-Jörg Kreowski, and Grzegorz Rozenberg, editors, Graph Transformation, First International Conference, ICGT 2002, Barcelona, Spain, October 7-12, 2002, Proceedings, volume 2505 of Lecture Notes in Computer Science, pages 161-176. Springer, 2002.

[HS01] Reiko Heckel and Stefan Sauer. Strengthening uml collaboration diagrams by state transformations. In Heinrich Hussmann, editor, Fundamental Approaches to Software Engineering, 4th International Conference, FASE 2001, held as Part of the Joint European Conferences on Theory and Practice of Software, ETAPS 2001, Genova, Italy, April 2-6, 2001, Proceedings, volume 2029 of LNCS, pages 109-123. Springer, 2001.

[KC00] Soon-Kyeong Kim and David Carrington. An integrated framework with UML and Object-Z for developing a precise specification. In N.N., editor, Proceedings of APSEC 2000. IEEE Computer Society, 2000.

[KGKK02] Sabine Kuske, Martin Gogolla, Ralf Kollmann, and Hans-Jörg Kreowski. An Integrated Semantics for UML Class, Object, and State Diagrams based on Graph Transformation. In Michael Butler and Kaisa Sere, editors, 3rd Int. Conf. Integrated Formal Methods (IFM'O2), volume 2335 of LNCS, pages 11-28. Springer, 2002.

[KK99] Hans-Jörg Kreowski and Sabine Kuske. Graph transformation units with interleaving semantics. Formal Aspects of Computing, 11(6):690-723, 1999.

[Kus00] Sabine Kuske. Transformation Units-A structuring Principle for Graph Transformation Systems. PhD thesis, University of Bremen, 2000.

[Kus01] Sabine Kuske. A formal semantics of uml state machines based on structured graph transformation. In Martin Gogolla and Cris Kobryn, editors, UML 2001 - The Unifi ed Modeling Language. Modeling Languages, Concepts, and Tools, volume 2185 of Lecture Notes in Computer Science, pages 241-256, 2001.

[OMG03] OMG. OMG Unified Modeling Language Specification, Version 1.5, March 2003. Object Management Group, Inc., Framingham, Mass., http://www.omg.org, 2003.

[Ric02] Mark Richters. A Precise Approach to Validating UML Models and OCL Constraints. $\mathrm{PhD}$ thesis, Universität Bremen, Logos Verlag, Berlin, BISS Monographs, No. 14, 2002. 
[Roz97] Grzegorz Rozenberg, editor. Handbook of Graph Grammars and Computing by Graph Transformation, Vol. 1: Foundations. World Scientific, Singapore, 1997.

[SCH01] Wuwei Shen, Kevin J. Compton, and James Huggins. A UML Validation Toolset Based on Abstract State Machines. In ASE, pages 315-318. IEEE Computer Society, 2001.

[SCH03] Wuwei Shen, Kevin J. Compton, and James Huggins. A Method of Implementing UML Virtual Machines With Some Constraints Based on Abstract State Machines. In APSEC, pages 224-232. IEEE Computer Society, 2003.

[SS00] Shane Sendall and Alfred Strohmeier. From use cases to system operation specifications. In Andy Evans, Stuart Kent, and Bran Selic, editors, UML 2000 - The Unifi ed Modeling Language. Advancing the Standard. Third International Conference, York, UK, October 2000, Proceedings, volume 1939 of LNCS, pages 1-15. Springer, 2000.

[Sta01] Leon Starr. Executable Uml: How to Build Class Models. Prentice Hall PTR, Upper Saddle River, NJ, USA, 2001. Foreword By-Stephen J. Mellor.

[Stö05] Harald Störrle. Semantics and Verification of Data Flow in UML 2.0 Activities. In Mark Minas, editor, Proceedings of the Workshop on Visual Languages and Formal Methods (VLFM 2004), volume 127(4) of Electronic Notes in Theoretical Computer Science, pages 35-52. Elsevier Science, 2005.

[TE00] A. Tsiolakis and H. Ehrig. Consistency analysis of UML class and sequence diagrams using attributed graph grammars. In H. Ehrig and G. Taentzer, editors, Proc. of Joint APPLIGRAPH/GETGRATS Workshop on Graph Transformation Systems, Berlin, March 2000, 2000. Technical Report no. 2000/2, Technical University of Berlin.

[USE05] A UML-based Specification Environment, last revision 2005. http://www.db.informatik.uni-bremen.de/projects/USE.

[Var02] Dániel Varró. A formal semantics of UML statecharts by model transition systems. In Andrea Corradini, Hartmut Ehrig, Hans-Jörg Kreowski, and Grzegorz Rozenberg, editors, Graph Transformation. First International Conference, ICGT 2002, Barcelona, Spain, October 2002, Proceedings, volume 2505 of LNCS, pages 378-392. Springer, 2002.

[Wie98] Roel Wieringa. Formalizing the UML in a systems engineering approach. In Haim Kilov and Bernhard Rumpe, editors, Proceedings Second ECOOP Workshop on Precise Behavioral Semantics (with an Emphasis on OO Business Specifi cations), pages 254-266. Technische Universität München, TUM-I9813, 1998.

[Zie05] Paul Ziemann. An Integrated Operational Semantics for a UML Core Based on Graph Transformation. PhD thesis, University of Bremen, 2005. 